\title{
THE COMPLETE ELLIPSOIDAL SHELL-MODEL IN EEG IMAGING
}

\author{
S. N. GIAPALAKI AND F. KARIOTOU
}

Received 5 December 2004; Accepted 16 December 2004

This work provides the solution of the direct Electroencephalography (EEG) problem for the complete ellipsoidal shell-model of the human head. The model involves four confocal ellipsoids that represent the successive interfaces between the brain tissue, the cerebrospinal fluid, the skull, and the skin characterized by different conductivities. The electric excitation of the brain is due to an equivalent electric dipole, which is located within the inner ellipsoid. The proposed model is considered to be physically complete, since the effect of the substance surrounding the brain is taken into account. The direct EEG problem consists in finding the electric potential inside each conductive space, as well as at the nonconductive exterior space. The solution of this multitransmission problem is given analytically in terms of elliptic integrals and ellipsoidal harmonics, in such way that makes clear the effect that each shell has on the next one and outside of the head. It is remarkable that the dependence on the observation point is not affected by the presence of the conductive shells. Reduction to simpler ellipsoidal models and to the corresponding spherical models is included.

Copyright (c) 2006 S. N. Giapalaki and F. Kariotou. This is an open access article distributed under the Creative Commons Attribution License, which permits unrestricted use, distribution, and reproduction in any medium, provided the original work is properly cited.

\section{Introduction}

The method of Electroencephalography (EEG) is the most widely used, noninvasive method for studying the human brain in vivo. The data of an Electroencephalogram are obtained by measuring the electric potentials in the exterior of the head. The inverse EEG problem consists in determining the location of the electrochemical source inside the brain that produces the externally measured electric potential field. The results obtained from the solution of the forward EEG problem, namely the electric potential field that a given source produces, are of major importance for the inverse problem. The 
well-poseness of the mathematical problem demands certain assumptions concerning the physical model approximating the electrochemical source as well as the geometrical model used for the brain-head approximation. The most popular model used for the source is that of an equivalent electric dipole current of a given moment.

As far as the geometrical model of the conductor is concerned and for the analytical treatment of the problem, the dominant model for the brain-head system is the one of a homogeneous spherical $[3,6,2,13]$, or a homogeneous spheroidal $[2,16]$ conductor. The improvement of these models, so that the $3 \mathrm{D}$ anisotropy of the system is taken into account, has led to the concept of more realistic volume conductors [12]. Furthermore, the case of a homogeneous ellipsoidal conductor, which fits best to the geometrical characteristics of the brain [14], was treated in [8]. Furthermore, the brain is protected by shells consisted of the cerebrospinal fluid, the bone and the skin that are all characterized by different electrical conductivities. As it is expected, this inhomogeneity constitutes an important parameter of the problem that should be taken into account considering layered volume conductors [2]. The case of one confocal ellipsoidal shell, characterised by different conductivity, surrounding the homogeneous ellipsoidal conductor, represents the brain was treated in [7].

In this work, in order to study the effect of inhomogeneity in the measured electric potential for the case of ellipsoidal geometry, we assume the physically complete ellipsoidal shell-model. Specifically we consider three confocal ellipsoidal shells, each one characterized by a different conductivity, which surround the homogeneous ellipsoidal conductor representing the brain. It is observed that the conductivity values, as well as the geometrical parameters of the four ellipsoidal boundaries, appear in every term of the multipole expansion of the electric potential, justifying this way the improvement offered by this model.

The postulation of the transmission problem that the electric potential field has to satisfy near the dipole source, in the spaces between the ellipsoidal boundaries and in the exterior space, is presented in Section 2. In Section 3 we deal with the solution of this problem using eigenfunction expansions in ellipsoidal coordinates. The solution is expressed in terms of elliptic integrals and ellipsoidal harmonics, while relative expressions in Cartesian and in tensorial form are also included. In Section 4 the corresponding homogeneous and one shell inhomogeneous ellipsoidal results are recovered through reduction process and also the electric potential for the two confocal ellipsoidal shell model is provided. The corresponding manipulations needed for the reduction to the sphericalshell model are given in Section 5.

\section{Statement of the problem}

Following anatomic structure we model the head as an ellipsoid, occupied by the brain, which is surrounded by three confocal ellipsoidal shells, which are filled, starting from the inside, with the cerebrospinal fluid, the skull and the skin. From the physical point of view the above compartments of this realistic model of the head are distinguished by their different values of electric conductivity. 
Let $S_{s}, S_{b}, S_{f}$, and $S_{c}$ denote the triaxial ellipsoidal surfaces, which in rectangular coordinates are specified by

$$
\begin{array}{ll}
\frac{x_{1}^{2}}{s_{1}^{2}}+\frac{x_{2}^{2}}{s_{2}^{2}}+\frac{x_{3}^{2}}{s_{3}^{2}}=1, & 0<s_{3}<s_{2}<s_{1}<+\infty, \\
\frac{x_{1}^{2}}{b_{1}^{2}}+\frac{x_{2}^{2}}{b_{2}^{2}}+\frac{x_{3}^{2}}{b_{3}^{2}}=1, & 0<b_{3}<b_{2}<b_{1}<+\infty, \\
\frac{x_{1}^{2}}{f_{1}^{2}}+\frac{x_{2}^{2}}{f_{2}^{2}}+\frac{x_{3}^{2}}{f_{3}^{2}}=1, & 0<f_{3}<f_{2}<f_{1}<+\infty, \\
\frac{x_{1}^{2}}{c_{1}^{2}}+\frac{x_{2}^{2}}{c_{2}^{2}}+\frac{x_{3}^{2}}{c_{3}^{2}}=1, & 0<c_{3}<c_{2}<c_{1}<+\infty,
\end{array}
$$

respectively, where $c_{i}<f_{i}<b_{i}<s_{i}, i=1,2,3$, are their semiaxes. The ellipsoids (2.1), (2.2), (2.3), (2.4) are confocal and correspond to the ellipsoidal system $\rho, \mu, \nu$ [5] with semifocal distances $h_{1}, h_{2}, h_{3}$, where

$$
\begin{aligned}
& h_{1}^{2}=s_{2}^{2}-s_{3}^{2}=b_{2}^{2}-b_{3}^{2}=f_{2}^{2}-f_{3}^{2}=c_{2}^{2}-c_{3}^{2}, \\
& h_{2}^{2}=s_{1}^{2}-s_{3}^{2}=b_{1}^{2}-b_{3}^{2}=f_{1}^{2}-f_{3}^{2}=c_{1}^{2}-c_{3}^{2}, \\
& h_{3}^{2}=s_{1}^{2}-s_{2}^{2}=b_{1}^{2}-b_{2}^{2}=f_{1}^{2}-f_{2}^{2}=c_{1}^{2}-c_{2}^{2} .
\end{aligned}
$$

The ellipsoidal coordinates $\rho, \mu, \nu$ are connected to the Cartesian ones $x_{1}, x_{2}, x_{3}$ by the relations [5]

$$
\begin{gathered}
x_{1}=\frac{\rho \mu \nu}{h_{2} h_{3}}, \\
x_{2}=\frac{\sqrt{\rho^{2}-h_{3}^{2}} \sqrt{\mu^{2}-h_{3}^{2}} \sqrt{h_{3}^{2}-\nu^{2}}}{h_{1} h_{3}}, \\
x_{3}=\frac{\sqrt{\rho^{2}-h_{2}^{2}} \sqrt{h_{2}^{2}-\mu^{2}} \sqrt{h_{2}^{2}-\nu^{2}}}{h_{1} h_{2}},
\end{gathered}
$$

and vary in the intervals $\left[h_{2},+\infty\right),\left[h_{3}, h_{2}\right]$, and $\left[-h_{3}, h_{3}\right]$, respectively.

In terms of the variable $\rho$, the surfaces $S_{s}, S_{b}, S_{f}$, and $S_{c}$ correspond to $\rho=s_{1}, \rho=b_{1}$, $\rho=f_{1}$, and $\rho=c_{1}$ and represent the boundaries of the skin, the scull (bone), the fluid and the cerebrum, respectively. The interior to $S_{c}$ space $V_{c}$ corresponds to the interval $\rho \in\left[h_{2}, c_{1}\right)$ and is characterized by the conductivity $\sigma_{c}$. The ellipsoidal shell between $S_{c}$ and $S_{f}$, denoted by $V_{f}$, corresponds to the interval $\rho \in\left(c_{1}, f_{1}\right)$ and is characterized by the conductivity $\sigma_{f}$. The ellipsoidal shell between $S_{f}$ and $S_{b}$, denoted by $V_{b}$, corresponds to the interval $\rho \in\left(f_{1}, b_{1}\right)$ and is characterized by the conductivity $\sigma_{b}$. Finally, the ellipsoidal shell bounded by $S_{b}$ and $S_{s}$, is denoted by $V_{s}$, corresponds to the interval $\rho \in\left(b_{1}, s_{1}\right)$ and is characterized by the conductivity $\sigma_{s}$. The exterior to $S_{s}$ nonconductive space $V$ is described by $\rho \in\left(s_{1},+\infty\right)$. 
4 The complete ellipsoidal shell-model in EEG imaging

At the point $\mathbf{r}_{0} \in V_{c}$ there exists a primary current dipole source with moment $\mathbf{Q}$. This is specified by the current density function

$$
\mathbf{J}^{P}(\mathbf{r})=\mathbf{Q} \delta\left(\mathbf{r}-\mathbf{r}_{0}\right)
$$

where $\delta$ stands for the Dirac measure at the point $\mathbf{r}_{0}$.

The primary current $\mathbf{J}^{P}$ induces an electric field $\mathbf{E}$ in the interior conductive space, which in turn generates an induction current with density $\mathrm{J}^{V}$ :

$$
\mathbf{J}^{V}(\mathbf{r})=\sigma_{c} \mathbf{E}_{c}(\mathbf{r}) X_{V_{c}}(\mathbf{r})+\sigma_{f} \mathbf{E}_{f}(\mathbf{r}) X_{V_{f}}(\mathbf{r})+\sigma_{b} \mathbf{E}_{b}(\mathbf{r}) X_{V_{b}}(\mathbf{r})+\sigma_{s} \mathbf{E}_{s}(\mathbf{r}) X_{V_{s}}(\mathbf{r}),
$$

where $X_{A}(\mathbf{r})$ denotes the characteristic function of the set $A$.

Hence, the total current at every point $\mathbf{r}$ of the conductor is given by

$$
\mathbf{J}(\mathbf{r})=\mathbf{J}^{P}(\mathbf{r})+\mathbf{J}^{V}(\mathbf{r})
$$

The current J generates an electromagnetic field, which propagates in the interior as well as in the exterior of the conductive space.

Because of the values of the dielectric constant and the electric conductivity of the brain tissue, the quasistatic approximation of Maxwell's equations is considered $[4,9,13$, 15]. Therefore the electric field $\mathbf{E}$ and the magnetic induction field $\mathbf{B}$ satisfy the following equations [9]:

$$
\begin{aligned}
\nabla \times \mathbf{E} & =\mathbf{0}, \\
\nabla \times \mathbf{B} & =\mu_{0} \mathbf{J}, \\
\nabla \cdot \mathbf{E} & =0, \\
\nabla \cdot \mathbf{B} & =0,
\end{aligned}
$$

where $\mu_{0}$ denotes the magnetic permeability in the whole space.

Since $\mathrm{E}$ is irrotational, it can be represented by an electric potential $u$, via the differential representation

$$
\mathbf{E}(\mathbf{r})=-\nabla u(\mathbf{r})
$$

The electric potential $u$ is the field recorded in any electroencephalogram. In particular, we denote the electric potential in the interior space $V_{c}$ by $u_{c}$, in the ellipsoidal shell $V_{f}$ by $u_{f}$, in the ellipsoidal shell $V_{b}$ by $u_{b}$, in the ellipsoidal shell $V_{s}$ by $u_{s}$ and in the exterior space $V$ by $u$. Combining (2.9), (2.14), and (2.11), we obtain the Poisson equation

$$
\Delta u_{c}(\mathbf{r})=\frac{1}{\sigma_{c}} \nabla \cdot \mathbf{J}^{P}(\mathbf{r}), \quad \mathbf{r} \in V_{c},
$$

which the interior potential $u_{c}$ must satisfy in $V_{c}$. 
In the source-free spaces $V_{f}, V_{b}, V_{s}$, and $V$ the potentials $u_{f}, u_{b}, u_{s}$, and $u$ solve the Laplace equation

$$
\begin{array}{cl}
\Delta u_{f}(\mathbf{r})=0, & \mathbf{r} \in V_{f}, \\
\Delta u_{b}(\mathbf{r})=0, & \mathbf{r} \in V_{b}, \\
\Delta u_{s}(\mathbf{r})=0, & \mathbf{r} \in V_{s}, \\
\Delta u(\mathbf{r})=0, & \mathbf{r} \in V .
\end{array}
$$

On the surface $S_{c}$ the following transmission conditions hold

$$
\begin{gathered}
u_{f}(\mathbf{r})=u_{c}(\mathbf{r}), \quad \mathbf{r} \in S_{c}, \\
\sigma_{f} \partial_{n} u_{f}(\mathbf{r})=\sigma_{c} \partial_{n} u_{c}(\mathbf{r}), \quad \mathbf{r} \in S_{c},
\end{gathered}
$$

where the $\partial_{n}$ indicates the outward normal differentiation. Conditions (2.20)-(2.21) state the continuity of the potential function as well as the continuity of the normal component of the electric field on $S_{c}$.

On the surface $S_{f}$ we demand that

$$
\begin{aligned}
u_{b}(\mathbf{r}) & =u_{f}(\mathbf{r}), \quad \mathbf{r} \in S_{f}, \\
\sigma_{b} \partial_{n} u_{b}(\mathbf{r}) & =\sigma_{f} \partial_{n} u_{f}(\mathbf{r}), \quad \mathbf{r} \in S_{f}
\end{aligned}
$$

and similarly on $S_{b}$,

$$
\begin{aligned}
u_{b}(\mathbf{r}) & =u_{s}(\mathbf{r}), \quad \mathbf{r} \in S_{b}, \\
\sigma_{b} \partial_{n} u_{b}(\mathbf{r}) & =\sigma_{s} \partial_{n} u_{s}(\mathbf{r}), \quad \mathbf{r} \in S_{b} .
\end{aligned}
$$

Since $V$ is characterized by zero conductivity, on the surface $S_{s}$ the continuity conditions read

$$
\begin{array}{ll}
u_{s}(\mathbf{r})=u(\mathbf{r}), & \mathbf{r} \in S_{s}, \\
\partial_{n} u_{s}(\mathbf{r})=0, & \mathbf{r} \in S_{s} .
\end{array}
$$

In addition the asymptotic behavior at infinity

$$
u(\mathbf{r})=O\left(\frac{1}{r}\right), \quad r \longrightarrow \infty
$$

has to be imposed in order to insure uniqueness.

\section{The interior and exterior electric potential}

The basic notation for the spectral decomposition of the Laplace operator in ellipsoidal coordinates can be found in $[1,5,7,8]$, where all interior $\mathbb{E}_{n}^{m}(\rho, \mu, \nu)$ and exterior $\mathbb{F}_{n}^{m}(\rho, \mu$, $\nu$ ) ellipsoidal harmonics that are used in this work, as well as useful relations connecting them, can be found. We recall the definition

$$
\mathbb{F}_{n}^{m}(\rho, \mu, \nu)=(2 n+1) I_{n}^{m}(\rho) \mathbb{E}_{n}^{m}(\rho, \mu, \nu)=(2 n+1) I_{n}^{m}(\rho) E_{n}^{m}(\rho) E_{n}^{m}(\mu) E_{n}^{m}(\nu)
$$


6 The complete ellipsoidal shell-model in EEG imaging

which connects the ellipsoidal exterior harmonics $\mathbb{F}_{n}^{m}(\mathbf{r})$ to the interior ellipsoidal harmonics $\mathbb{E}_{n}^{m}(\mathbf{r})$ via the elliptic integrals

$$
I_{n}^{m}(\rho)=\int_{\rho}^{\infty} \frac{d t}{\left[E_{n}^{m}(t)\right]^{2} \sqrt{t^{2}-h_{2}^{2}} \sqrt{t^{2}-h_{3}^{2}}}
$$

where $E_{n}^{m}(x)$ are the Lamé functions of the first kind.

The solution of (2.19), is an exterior harmonic function which assumes the exterior ellipsoidal expansion

$$
u(\rho, \mu, \nu)=\sum_{n=0}^{\infty} \sum_{m=1}^{2 n+1} f_{n}^{m} \mathbb{F}_{n}^{m}(\rho, \mu, \nu), \quad \rho>s_{1},
$$

and satisfies automatically the asymptotic condition (2.28).

Inside the ellipsoidal shells $V_{s}, V_{b}, V_{f}$ the electric potentials $u_{s}, u_{b}, u_{f}$ solve (2.18), (2.17), (2.16), respectively, and therefore they assume the following ellipsoidal expansions

$$
\begin{array}{ll}
u_{s}(\mathbf{r})=\sum_{n=0}^{\infty} \sum_{m=1}^{2 n+1}\left[g_{n}^{m} \mathbb{E}_{n}^{m}(\rho, \mu, \nu)+h_{n}^{m} \mathbb{F}_{n}^{m}(\rho, \mu, \nu)\right], & b_{1}<\rho<s_{1}, \\
u_{b}(\mathbf{r})=\sum_{n=0}^{\infty} \sum_{m=1}^{2 n+1}\left[k_{n}^{m} \mathbb{E}_{n}^{m}(\rho, \mu, \nu)+m_{n}^{m} \mathbb{F}_{n}^{m}(\rho, \mu, \nu)\right], & f_{1}<\rho<b_{1}, \\
u_{f}(\mathbf{r})=\sum_{n=0}^{\infty} \sum_{m=1}^{2 n+1}\left[p_{n}^{m} \mathbb{E}_{n}^{m}(\rho, \mu, \nu)+q_{n}^{m} \mathbb{F}_{n}^{m}(\rho, \mu, \nu)\right] & c_{1}<\rho<f_{1} .
\end{array}
$$

Finally, in the interior space $V_{c}$, which includes the primary source $\mathbf{J}^{p}$, the interior electric potential $u_{b}$ solves (2.15), and it is given as a superposition of an interior harmonic function $\Phi(\mathbf{r})$ and the particular solution of Poisson's equation

$$
V(\mathbf{r})=-\frac{1}{4 \pi \sigma_{c}} \mathbf{Q} \cdot \nabla_{\mathbf{r}} \frac{1}{\left|\mathbf{r}-\mathbf{r}_{0}\right|}=\frac{1}{4 \pi \sigma_{c}} \mathbf{Q} \cdot \nabla_{\mathbf{r}_{0}} \frac{1}{\left|\mathbf{r}-\mathbf{r}_{0}\right|} .
$$

Using the ellipsoidal expansion for the interior harmonic function $\Phi(\mathbf{r})$,

$$
\Phi(\mathbf{r})=\sum_{n=0}^{\infty} \sum_{m=1}^{2 n+1} t_{n}^{m} \mathbb{E}_{n}^{m}(\rho, \mu, v)
$$

we can write the interior electric potential as

$$
u_{c}(\mathbf{r})=\frac{1}{4 \pi \sigma_{c}} \mathbf{Q} \cdot \nabla_{\mathbf{r}_{0}} \frac{1}{\left|\mathbf{r}-\mathbf{r}_{0}\right|}+\sum_{n=0}^{\infty} \sum_{m=1}^{2 n+1} t_{n}^{m} \mathbb{E}_{n}^{m}(\rho, \mu, \nu), \quad \rho<c_{1} .
$$

The ellipsoidal expansion of the fundamental solution of the Laplace operator for $\rho>\rho_{0}$ is given in [11] by

$$
\frac{1}{\left|\mathbf{r}-\mathbf{r}_{0}\right|}=\sum_{n=0}^{\infty} \sum_{m=1}^{2 n+1} \frac{4 \pi}{2 n+1} \frac{1}{\gamma_{n}^{m}} \mathbb{E}_{n}^{m}\left(\rho_{0}, \mu_{0}, \nu_{0}\right) \mathbb{F}_{n}^{m}(\rho, \mu, \nu),
$$


where $\gamma_{n}^{m}$ are the normalization constants of the surface ellipsoidal harmonics. Applying properly the gradient operator on (3.8), we obtain the following form for $u_{c}$ :

$$
u_{c}(\mathbf{r})=t_{0}^{1}+\sum_{n=1}^{\infty} \sum_{m=1}^{2 n+1}\left[t_{n}^{m}+\frac{1}{\sigma_{c} \gamma_{n}^{m}}\left(\mathbf{Q} \cdot \nabla_{\mathbf{r}_{0}} \mathbb{E}_{n}^{m}\left(\rho_{0}, \mu_{0}, \nu_{0}\right)\right) I_{n}^{m}(\rho)\right] \mathbb{E}_{n}^{m}(\rho, \mu, \nu) .
$$

In (3.9) we have further expressed the exterior ellipsoidal harmonics in terms of the corresponding interior ones, by means of the elliptic integral $I_{n}^{m}$. Expansion (3.9) holds for $\rho>\rho_{0}$, therefore it holds true on all boundaries $S_{c}, S_{f}, S_{b}, S_{s}$. In (3.3), (3.4) and (3.9) we have expressed all the potentials in terms of ellipsoidal harmonics and therefore the application of the transmission conditions (2.20)-(2.27) is straightforward. Furthermore, the homogeneity of (2.21), (2.23), (2.25), and (2.27) in the operator $\partial_{n}$ allows for the replacement of the normal derivative $\partial_{n}$ with the $\rho$-derivative $\partial_{\rho}$, since the corresponding metric coefficient cancels out.

Introducing (3.3), (3.4), and (3.9) in the boundary conditions (2.20)-(2.27) and using the orthogonality property of the surface ellipsoidal harmonics, the constants $f_{n}^{m}, g_{n}^{m}, h_{n}^{m}$, $k_{n}^{m}, m_{n}^{m}, p_{n}^{m}, q_{n}^{m}, t_{n}^{m}$ are determined as the solutions of a $8 \times 8$ linear algebraic system. Long but straightforward calculations, which are not shown here, lead to the expressions:

$$
\begin{gathered}
g_{0}^{1}=k_{0}^{1}=p_{0}^{1}=t_{0}^{1}=f_{0}^{1} I_{0}^{1}\left(s_{1}\right), \\
h_{0}^{1}=m_{0}^{1}=q_{0}^{1}=0
\end{gathered}
$$

while for $n=1,2, \ldots, m=1,2, \ldots, 2 n+1$, particular expressions for the eight sequences of constants $f_{n}^{m}, g_{n}^{m}, h_{n}^{m}, k_{n}^{m}, l_{n}^{m}, p_{n}^{m}, q_{n}^{m}, t_{n}^{m}$, which contain the Lamé functions of the first and the second kind evaluated at specific points are obtained. Introducing the notation

$$
\begin{aligned}
I_{n}^{m}(x, y)=I_{n}^{m}(x)-I_{n}^{m}(y)=\int_{x}^{y} \frac{d t}{\left[E_{n}^{m}(t)\right]^{2} \sqrt{t^{2}-h_{2}^{2}} \sqrt{t^{2}-h_{3}^{2}}}, \\
S_{n}^{m}=E_{n}^{m}\left(s_{1}\right) E_{n}^{m^{\prime}}\left(s_{1}\right) s_{2} s_{3}, \\
B_{n}^{m}=E_{n}^{m}\left(b_{1}\right) E_{n}^{m^{\prime}}\left(b_{1}\right) b_{2} b_{3}, \\
F_{n}^{m}=E_{n}^{m}\left(f_{1}\right) E_{n}^{m^{\prime}}\left(f_{1}\right) f_{2} f_{3}, \\
C_{n}^{m}=E_{n}^{m}\left(c_{1}\right) E_{n}^{m^{\prime}}\left(c_{1}\right) c_{2} c_{3},
\end{aligned}
$$

where the prime denotes differentiation with respect to the variable and using the corresponding values of the coefficients in (3.3), (3.4), and (3.9) we obtain the following expressions for the potential fields $u, u_{s}, u_{b}, u_{f}$, and $u_{c}$ which hold true in the indicated regions. In particular for the exterior space we obtain

$$
u(\mathbf{r})=g_{0}^{1} \frac{I_{0}^{1}(\rho)}{I_{0}^{1}\left(s_{1}\right)}+\sum_{n=1}^{\infty} \sum_{m=1}^{2 n+1} \frac{I_{n}^{m}(\rho)}{I_{n}^{m}\left(s_{1}\right)} \frac{1}{S_{n}^{m}} \frac{1}{G_{3, n}^{m}} \frac{\mathbf{Q} \cdot \nabla \mathbb{E}_{n}^{m}\left(\mathbf{r}_{0}\right)}{\gamma_{n}^{m}} \mathbb{E}_{n}^{m}(\rho, \mu, \nu), \quad \rho>s_{1},
$$


8 The complete ellipsoidal shell-model in EEG imaging

where $g_{0}^{1}$ is an arbitrary constant. For the skin region we obtain

$$
u_{s}(\mathbf{r})=u\left(s_{1}\right)+\sum_{n=1}^{\infty} \sum_{m=1}^{2 n+1} I_{n}^{m}\left(\rho, s_{1}\right) \frac{1}{G_{3, n}^{m}} \frac{\mathbf{Q} \cdot \nabla \mathbb{E}_{n}^{m}\left(\mathbf{r}_{0}\right)}{\gamma_{n}^{m}} \mathbb{E}_{n}^{m}(\rho, \mu, \nu), \quad b_{1}<\rho<s_{1},
$$

for the scull region

$$
u_{b}(\mathbf{r})=u_{s}\left(b_{1}\right)+\sum_{n=1}^{\infty} \sum_{m=1}^{2 n+1} I_{n}^{m}\left(\rho, b_{1}\right) \frac{1}{\sigma_{b}} \frac{G_{1, n}^{m}}{G_{3, n}^{m}} \frac{\mathbf{Q} \cdot \nabla \mathbb{E}_{n}^{m}\left(\mathbf{r}_{0}\right)}{\gamma_{n}^{m}} \mathbb{E}_{n}^{m}(\rho, \mu, \nu), \quad f_{1}<\rho<b_{1},
$$

for the fluid region

$$
u_{f}(\mathbf{r})=u_{b}\left(f_{1}\right)+\sum_{n=1}^{\infty} \sum_{m=1}^{2 n+1} I_{n}^{m}\left(\rho, f_{1}\right) \frac{1}{\sigma_{f}} \frac{G_{2, n}^{m}}{G_{3, n}^{m}} \frac{\mathbf{Q} \cdot \nabla \mathbb{E}_{n}^{m}\left(\mathbf{r}_{0}\right)}{\gamma_{n}^{m}} \mathbb{E}_{n}^{m}(\rho, \mu, \nu), \quad c_{1}<\rho<f_{1},
$$

and finally for the region occupied by the cerebrum we obtain

$$
u_{c}(\mathbf{r})=u_{f}\left(c_{1}\right)+\sum_{n=1}^{\infty} \sum_{m=1}^{2 n+1} I_{n}^{m}\left(\rho, c_{1}\right) \frac{1}{\sigma_{c}} \frac{\mathbf{Q} \cdot \nabla \mathbb{E}_{n}^{m}\left(\mathbf{r}_{0}\right)}{\gamma_{n}^{m}} \mathbb{E}_{n}^{m}(\rho, \mu, \nu), \quad \rho<c_{1} .
$$

The constants $G_{1, n}^{m}, G_{2, n}^{m}$ and $G_{3, n}^{m}$ are given by

$$
\begin{aligned}
G_{1, n}^{m}= & \sigma_{b}+\left(\sigma_{b}-\sigma_{s}\right)\left(I_{n}^{m}\left(b_{1}, s_{1}\right)+\frac{1}{S_{n}^{m}}-\frac{1}{B_{n}^{m}}\right) B_{n}^{m}, \\
G_{2, n}^{m}= & \sigma_{f}+\left(\sigma_{f}-\sigma_{b}\right)\left(I_{n}^{m}\left(f_{1}, s_{1}\right)+\frac{1}{S_{n}^{m}}-\frac{1}{F_{n}^{m}}\right) F_{n}^{m}+\left(\sigma_{b}-\sigma_{s}\right)\left(I_{n}^{m}\left(b_{1}, s_{1}\right)+\frac{1}{S_{n}^{m}}-\frac{1}{B_{n}^{m}}\right) B_{n}^{m} \\
& +\frac{\left(\sigma_{f}-\sigma_{b}\right)\left(\sigma_{b}-\sigma_{s}\right)}{\sigma_{b}} I_{n}^{m}\left(f_{1}, b_{1}\right)\left(I_{n}^{m}\left(b_{1}, s_{1}\right)+\frac{1}{S_{n}^{m}}-\frac{1}{B_{n}^{m}}\right) B_{n}^{m} F_{n}^{m}, \\
G_{3, n}^{m}= & \sigma_{c}+\left(\sigma_{c}-\sigma_{f}\right)\left(I_{n}^{m}\left(c_{1}, s_{1}\right)+\frac{1}{S_{n}^{m}}-\frac{1}{C_{n}^{m}}\right) C_{n}^{m} \\
& +\left(\sigma_{f}-\sigma_{b}\right)\left(I_{n}^{m}\left(f_{1}, s_{1}\right)+\frac{1}{S_{n}^{m}}-\frac{1}{F_{n}^{m}}\right) F_{n}^{m}+\left(\sigma_{b}-\sigma_{s}\right)\left(I_{n}^{m}\left(b_{1}, s_{1}\right)+\frac{1}{S_{n}^{m}}-\frac{1}{B_{n}^{m}}\right) B_{n}^{m} \\
& +\frac{\left(\sigma_{c}-\sigma_{f}\right)\left(\sigma_{f}-\sigma_{b}\right)}{\sigma_{f}} I_{n}^{m}\left(c_{1}, f_{1}\right)\left(I_{n}^{m}\left(f_{1}, s_{1}\right)+\frac{1}{S_{n}^{m}}-\frac{1}{F_{n}^{m}}\right) F_{n}^{m} C_{n}^{m} \\
& +\frac{\left(\sigma_{c}-\sigma_{f}\right)\left(\sigma_{b}-\sigma_{s}\right)}{\sigma_{b}} I_{n}^{m}\left(c_{1}, b_{1}\right)\left(I_{n}^{m}\left(b_{1}, s_{1}\right)+\frac{1}{S_{n}^{m}}-\frac{1}{B_{n}^{m}}\right) B_{n}^{m} C_{n}^{m} \\
& +\frac{\left(\sigma_{f}-\sigma_{b}\right)\left(\sigma_{b}-\sigma_{s}\right)}{\sigma_{b}} I_{n}^{m}\left(f_{1}, b_{1}\right)\left(I_{n}^{m}\left(b_{1}, s_{1}\right)+\frac{1}{S_{n}^{m}}-\frac{1}{B_{n}^{m}}\right) B_{n}^{m} F_{n}^{m} \\
& +\frac{\left(\sigma_{c}-\sigma_{f}\right)\left(\sigma_{f}-\sigma_{b}\right)\left(\sigma_{b}-\sigma_{s}\right)}{\sigma_{f} \sigma_{b}} \\
& \times I_{n}^{m}\left(c_{1}, f_{1}\right)\left(I_{n}^{m}\left(b_{1}, s_{1}\right)+\frac{1}{S_{n}^{m}}-\frac{1}{B_{n}^{m}}\right)\left(I_{n}^{m}\left(f_{1}, b_{1}\right)-\frac{1}{F_{n}^{m}}\right) B_{n}^{m} F_{n}^{m} C_{n}^{m} . \\
& \\
&
\end{aligned}
$$


In trying to interpret (3.16) to (3.20) we observe the following. Expression (3.16) provides the electric potential at any point outside the conductor. Then the potential within the outmost shell is expressed as the exterior potential $u$ evaluated on the surface $S_{s}$ of the skin, plus an expansion evaluated at the observation point $\mathbf{r}$, which represents the contribution that comes from the shell $V_{s}$. In a similar fashion, the potentials (3.17)-(3.19) within the following succesive shells, as well as the potential (3.20) inside the cerebrum region, are expressed as the potential of the exterior shell evaluated at their common boundary plus a contribution from the particular shell, always in the form of the appropriate eigenfunction expansion.

Furthermore, the form of each one of these expansions remains the same. They only differ by the constant ratios involving the conductivity profiles and by the fact that the corresponding elliptic integrals are evaluated on different surfaces. The above ratios specify the effect of the surrounding shells normalized by the effect of all shells considered in the model. Each ratio is multiplied by a conductivity factor which is what the equivalent homogeneous conductor would impose to the exterior electric potential.

It is worth noticing though that the part of the solution which is depended on the location of the observation point remains unaltered by the presence of the shells.

In the sequel we are going to work further on the expression (3.16), since the exterior potential is what it is registered on an electroencephalogram. Therefore, elaborating further on (3.16) by using the interior Lamé functions and the interior ellipsoidal harmonics in terms of the more tractable Cartesian coordinates and by calculating the action of the gradient on $\mathbb{E}_{n}^{m}$ and on $E_{n}^{m}$, we obtain the following analytic form of $u$ expressed in Cartesian coordinates and elliptic integrals

$$
\begin{aligned}
& u(\rho, \mu, \nu) \\
& =g_{0} \frac{I_{0}^{1}(\rho)}{I_{0}^{1}\left(s_{1}\right)}+\frac{3}{4 \pi s_{1} s_{2} s_{3}} \sum_{m=1}^{3} \frac{Q_{m} x_{m}}{G_{3,1}^{m}} \frac{I_{1}^{m}(\rho)}{I_{1}^{m}\left(s_{1}\right)} \\
& \quad-\frac{5}{8 \pi s_{1} s_{2} s_{3}\left(\Lambda_{s}-\Lambda_{s}^{\prime}\right)} \sum_{m=1}^{3} Q_{m} x_{0 m}\left[\frac{1}{G_{3,2}^{1}} \frac{I_{2}^{1}(\rho)}{I_{2}^{1}\left(s_{1}\right)} \frac{\mathbb{E}_{2}^{1}(\mathbf{r})}{\Lambda_{s}\left(\Lambda_{s}-s_{m}^{2}\right)}-\frac{1}{G_{3,2}^{2}} \frac{I_{2}^{2}(\rho)}{I_{2}^{2}\left(s_{1}\right)} \frac{\mathbb{E}_{2}^{2}(\mathbf{r})}{\Lambda_{s}^{\prime}\left(\Lambda_{s}^{\prime}-s_{m}^{2}\right)}\right] \\
& \quad+\frac{15}{4 \pi s_{1} s_{2} s_{3}} \sum_{\substack{i, j=1 \\
i \neq j}}^{3} \frac{Q_{i} x_{0 j} x_{i} x_{j}}{G_{3,2}^{6-i-j}\left(s_{i}^{2}+s_{j}^{2}\right)} \frac{I_{2}^{i+j}(\rho)}{I_{2}^{i+j}\left(s_{1}\right)}+O\left(\mathrm{el}_{3}\right) .
\end{aligned}
$$

The notation $O\left(\mathrm{el}_{3}\right)$ in (3.24) denotes ellipsoidal terms of degree greater or equal to three. The constants

$$
\left.\begin{array}{c}
\Lambda_{s} \\
\Lambda_{s}^{\prime}
\end{array}\right\}=s_{1}^{2}-\frac{1}{3}\left[h_{2}^{2}+h_{3}^{2} \pm \sqrt{h_{1}^{4}+h_{2}^{2} h_{3}^{2}}\right]
$$

satisfy the equation

$$
\sum_{m=1}^{3} \frac{1}{\Lambda_{s}-s_{m}^{2}}=0
$$


10 The complete ellipsoidal shell-model in EEG imaging

and generate the constant dyadics

$$
\begin{aligned}
& \tilde{\Lambda}_{s}=\frac{1}{G_{3,2}^{1}} \sum_{m=1}^{3} \frac{\hat{\mathbf{x}}_{m} \otimes \hat{\mathbf{x}}_{m}}{\Lambda_{s}-s_{m}^{2}}, \\
& \tilde{\Lambda}_{s}^{\prime}=\frac{1}{G_{3,2}^{2}} \sum_{m=1}^{3} \frac{\widehat{\mathbf{x}}_{m} \otimes \hat{\mathbf{x}}_{m}}{\Lambda_{s}^{\prime}-s_{m}^{2}} .
\end{aligned}
$$

Furthermore, in terms of the dyadic fields

$$
\begin{gathered}
\tilde{\mathbf{A}}(\rho)=\frac{3}{4 \pi s_{1} s_{2} s_{3}} \sum_{m=1}^{3} \frac{1}{G_{3,1}^{m}} \frac{I_{1}^{m}(\rho)}{I_{1}^{m}\left(s_{1}\right)} \hat{\mathbf{x}}_{m} \otimes \hat{\mathbf{x}}_{m}, \\
\widetilde{\mathbf{B}}(\mathbf{r})=-\frac{5}{8 \pi s_{1} s_{2} s_{3}\left(\Lambda_{s}-\Lambda_{s}^{\prime}\right)}\left[\frac{I_{2}^{1}(\rho)}{I_{2}^{1}\left(s_{1}\right)} \frac{\tilde{\Lambda}_{s}}{\Lambda_{s}} \mathbb{E}_{2}^{1}(\mathbf{r})-\frac{I_{2}^{2}(\rho)}{I_{2}^{2}\left(s_{1}\right)} \frac{\tilde{\Lambda}_{s}^{\prime}}{\Lambda_{s}^{\prime}} \mathbb{E}_{2}^{2}(\mathbf{r})\right]
\end{gathered}
$$

and the tetradic field

$$
\widetilde{\widetilde{\Gamma}}(\rho)=\frac{15}{4 \pi s_{1} s_{2} s_{3}} \sum_{\substack{i, j=1 \\ i \neq j}}^{3} \frac{1}{G_{3,2}^{6-i-j}} \frac{I_{2}^{i+j}(\rho)}{I_{2}^{i+j}\left(s_{1}\right)} \frac{\widehat{\mathbf{x}}_{i} \otimes \hat{\mathbf{x}}_{j} \otimes \widehat{\mathbf{x}}_{i} \otimes \widehat{\mathbf{x}}_{j}}{s_{i}^{2}+s_{j}^{2}}
$$

we rewrite the electric field as

$$
u(\mathbf{r})=g_{0}^{1} \frac{I_{0}^{1}(\rho)}{I_{0}^{1}\left(s_{1}\right)}+\mathbf{Q} \cdot \widetilde{\mathbf{A}} \cdot \mathbf{r}+\mathbf{Q} \otimes \mathbf{r}_{0}: \widetilde{\mathbf{B}}(\mathbf{r})+\mathbf{Q} \otimes \mathbf{r}_{0}: \widetilde{\widetilde{\Gamma}}(\rho): \mathbf{r} \otimes \mathbf{r}+O\left(\mathrm{el}_{3}\right)
$$

where the double contraction is defined by

$$
\mathbf{a} \otimes \mathbf{b}: \mathbf{c} \otimes \mathbf{d}=(\mathbf{a} \cdot \mathbf{c})(\mathbf{b} \cdot \mathbf{d})
$$

The use of the polyadic notation in expressing the exterior electric potential offers the advantage of a unified and compact form in which the source enters in a distinctive and clear way. In fact, the polyadic fields $\widetilde{\mathbf{A}}(\rho), \widetilde{\mathbf{B}}(\mathbf{r}), \widetilde{\widetilde{\Gamma}}(\rho)$ include all the geometric and physical characteristics of the conductor while the moment and position of the source is obtained from them via simple and double contraction.

\section{Physical degeneracies}

Our purpose here is to recover from results (3.16), (3.17), (3.18), (3.19), and (3.20) for the electric potential fields in the four compartment ellipsoidal model, the corresponding results for the one shell model [7]. In the notation of the present work the corresponding results read as

$$
u_{1}(\mathbf{r})=g_{0}^{1} \frac{I_{0}^{1}(\rho)}{I_{0}^{1}\left(s_{1}\right)}+\sum_{n=1}^{\infty} \sum_{m=1}^{2 n+1} \frac{I_{n}^{m}(\rho)}{I_{n}^{m}\left(s_{1}\right)} \frac{1}{S_{n}^{m}} \frac{1}{G_{1, n}^{m}} \frac{\mathbf{Q} \cdot \nabla \mathbb{E}_{n}^{m}\left(\mathbf{r}_{0}\right)}{\gamma_{n}^{m}} \mathbb{E}_{n}^{m}(\rho, \mu, \nu)
$$


for $\rho>s_{1}$,

$$
u_{1, s}(\mathbf{r})=u_{1}\left(s_{1}\right)+\sum_{n=1}^{\infty} \sum_{m=1}^{2 n+1} I_{n}^{m}\left(\rho, s_{1}\right) \frac{1}{G_{1, n}^{m}} \frac{\mathbf{Q} \cdot \nabla \mathbb{E}_{n}^{m}\left(\mathbf{r}_{0}\right)}{\gamma_{n}^{m}} \mathbb{E}_{n}^{m}(\rho, \mu, \nu)
$$

for $c_{1}<\rho<s_{1}$ and

$$
u_{1, c}(\mathbf{r})=u_{1, s}\left(c_{1}\right)+\sum_{n=1}^{\infty} \sum_{m=1}^{2 n+1} I_{n}^{m}\left(\rho, c_{1}\right) \frac{1}{\sigma_{c}} \frac{\mathbf{Q} \cdot \nabla \mathbb{E}_{n}^{m}\left(\mathbf{r}_{0}\right)}{\gamma_{n}^{m}} \mathbb{E}_{n}^{m}(\rho, \mu, \nu)
$$

for $\rho_{0}<\rho<c_{1}$, where $s_{1}, c_{1}$ appear in (2.1) and (2.4), respectively.

In (4.1), $g_{0}^{1}$ is an arbitrary constant and the rest of the notation in (4.1), (4.2), and (4.3) remain identical with the present work. In order to reduce the three shells-ellipsoidal model to the one shell-ellipsoidal model we need to unify appropriately the spaces $V_{s}, V_{b}$, $V_{f}$ and $V_{c}$. This is obtained by the following three options. One corresponds to taking the limits

$$
\sigma_{f} \longrightarrow \sigma_{b} \longrightarrow \sigma_{s}
$$

while the conductivity of the core remains $\sigma_{c}$.

The second choice corresponds to

$$
\sigma_{b} \longrightarrow \sigma_{s}, \quad \sigma_{f} \longrightarrow \sigma_{c}
$$

and the third one is obtained by

$$
\sigma_{b} \longrightarrow \sigma_{f} \longrightarrow \sigma_{c}
$$

while we preserve the conductivity of the outer boundary to be $\sigma_{s}$.

Whichever of these three settings we choose, the results for the one shell-ellipsoidal model are recovered. Indicatively we select the first alternative, which geometrically corresponds to

$$
f_{i} \longrightarrow b_{i} \longrightarrow s_{i}, \quad i=1,2,3 .
$$

and it is denoted by $3 \mathrm{sh} \rightarrow 1 \mathrm{sh}$. As a consequence, from (3.21) we obtain

$$
\lim _{3 \mathrm{sh} \rightarrow 1 \mathrm{sh}}\left(G_{1, n}^{m}\right)=\sigma_{s}
$$

while from (3.22) we obtain

$$
\lim _{3 \mathrm{sh} \rightarrow 1 \mathrm{sh}}\left(G_{2, n}^{m}\right)=\sigma_{s}
$$

and finally, in view of (3.23),

$$
\lim _{3 \mathrm{sh} \rightarrow 1 \mathrm{sh}}\left(G_{3, n}^{m}\right)=\sigma_{c}+\left(\sigma_{c}-\sigma_{s}\right)\left(I_{n}^{m}\left(c_{1}, s_{1}\right)+\frac{1}{S_{n}^{m}}-\frac{1}{C_{n}^{m}}\right) C_{n}^{m}
$$

which is the conductivity term for the one shell model. 
That explains the claim that $G_{3, n}^{m}$ plays the role of the conductivity term in the case of the one shell model, incorporating the geometry as well as the physics of the different conductivity supports. Under these reductions (3.17), (3.18), and (3.19) degenerate to

$$
\lim _{3 \mathrm{sh} \rightarrow 1 \mathrm{sh}} u_{f}(\mathbf{r})=\lim _{3 \mathrm{sh} \rightarrow 1 \mathrm{sh}} u_{b}(\mathbf{r})=\lim _{3 \mathrm{sh} \rightarrow 1 \mathrm{sh}} u_{s}(\mathbf{r})=u_{1, s}(\mathbf{r})
$$

and (3.16), (3.20) give immediately

$$
\lim _{3 \mathrm{sh} \rightarrow 1 \mathrm{sh}} u(\mathbf{r})=u_{1}(\mathbf{r}), \quad \lim _{3 \mathrm{sh} \rightarrow 1 \mathrm{sh}} u_{c}(\mathbf{r})=u_{1, c}(\mathbf{r})
$$

respectively.

Comparing results (3.16), (3.17), (3.18), (3.19), and (3.20) with (4.1), (4.2), and (4.3), it is obvious that the effect of the presence of the shell-inhomogeneity on the electric potential fields is incorporated into the conductivity factor multiplying each multipole term, leaving the rest of the structure invariant. This observation is enhanced in the sequel where we reduce further our model to the absence of any inhomogeneous shell covering the conductivity core. Actually, we notice that the exterior electric potential, in both the three-shells model and the one-shell model, enjoys the same structure. Moreover, all the effects of the three shells are incorporated in the conductivity factor multiplying each multipole term.

Afterwards, we recover from the corresponding results (3.16), (3.17), (3.18), (3.19), and (3.20) for the electric potential fields in the three-shells model, the results for the single model which is the case of a homogeneous ellipsoidal conductor characterized by conductivity $\sigma$. As it is shown in [8], using the notation of the present work, the exterior potential assumes the form

$$
u_{0}(\mathbf{r})=g_{0} \frac{I_{0}^{1}(\rho)}{I_{0}^{1}\left(s_{1}\right)}+\sum_{n=1}^{\infty} \sum_{m=1}^{2 n+1} \frac{I_{n}^{m}(\rho)}{I_{n}^{m}\left(s_{1}\right)} \frac{1}{S_{n}^{m}} \frac{1}{\sigma} \frac{\mathbf{Q} \cdot \nabla \mathbb{E}_{n}^{m}\left(\mathbf{r}_{0}\right)}{\gamma_{n}^{m}} \mathbb{E}_{n}^{m}(\rho, \mu, \nu)
$$

for $\rho>s_{1}$, while the interior potential is given by

$$
u_{0, s}(\mathbf{r})=u_{0}\left(s_{1}\right)+\sum_{n=1}^{\infty} \sum_{m=1}^{2 n+1} I_{n}^{m}\left(\rho, s_{1}\right) \frac{1}{\sigma} \frac{\mathbf{Q} \cdot \nabla \mathbb{E}_{n}^{m}\left(\mathbf{r}_{0}\right)}{\gamma_{n}^{m}} \mathbb{E}_{n}^{m}(\rho, \mu, \nu)
$$

for $\rho_{0}<\rho<s_{1}$.

In order to reduce the three-shells model to the homogeneous ellipsoidal conductor we need to unify the spaces $V_{s}, V_{b}, V_{f}$, and $V_{c}$. This is obtained by considering the limits

$$
\sigma_{c} \longrightarrow \sigma_{f} \longrightarrow \sigma_{b} \longrightarrow \sigma_{s}=\sigma
$$

which geometrically means that

$$
c_{i} \longrightarrow f_{i} \longrightarrow b_{i} \longrightarrow s_{i}, \quad i=1,2,3
$$

and it is denoted by $3 \mathrm{sh} \rightarrow$ hom. As a consequence, of (3.21), (3.22), and (3.23) we obtain

$$
\lim _{\text {3sh } \rightarrow \text { hom }}\left(G_{1, n}^{m}\right)=\lim _{\text {3sh } \rightarrow \text { hom }}\left(G_{2, n}^{m}\right)=\lim _{\text {3sh } \rightarrow \text { hom }}\left(G_{3, n}^{m}\right)=\sigma,
$$


where $\sigma$ is the constant conductivity of the homogeneous ellipsoidal conductor. That also justifies the claim that $G_{3, n}^{m}$ is reduced to the conductivity term in the case of the homogeneous ellipsoidal conductor.

Under these reductions (3.17), (3.18), (3.19), and (3.20) degenerate to

$$
\lim _{\text {3sh } \rightarrow \text { hom }} u_{c}(\mathbf{r})=\lim _{\text {3sh } \rightarrow \text { hom }} u_{f}(\mathbf{r})=\lim _{\text {3sh } \rightarrow \text { hom }} u_{b}(\mathbf{r})=\lim _{\text {3sh } \rightarrow \text { hom }} u_{s}(\mathbf{r})=u_{0, s}(\mathbf{r})
$$

and (3.16) implies immediately that

$$
\lim _{3 \text { sh } \rightarrow \text { hom }} u(\mathbf{r})=u_{0}(\mathbf{r})
$$

We turn now to the calculation of the electric potential fields in the case of the two-shells ellipsoidal model. This will be achieved by the reduction of the corresponding results for the 3-shells model, using appropriate settings for the conductivity profiles. As it is shown below, in the exterior potential form, the conductivity factor for this case is $G_{2, n}^{m}$, which has already been given in (3.22). Actually by setting

$$
\sigma_{c} \longrightarrow \sigma_{f}
$$

which geometrically means that

$$
c_{i} \longrightarrow f_{i}, \quad i=1,2,3
$$

and it is denoted by $3 \mathrm{sh} \rightarrow 2 \mathrm{sh}$, we observe that $G_{1, n}^{m}$ remains unaltered, as from (3.21) we obtain

$$
\lim _{3 \mathrm{sh} \rightarrow 2 \mathrm{sh}}\left(G_{1, n}^{m}\right)=\sigma_{b}+\left(\sigma_{b}-\sigma_{s}\right)\left(I_{n}^{m}\left(b_{1}, s_{1}\right)+\frac{1}{S_{n}^{m}}-\frac{1}{B_{n}^{m}}\right) B_{n}^{m}=G_{1, n}^{m} .
$$

Moreover (3.22) and (3.23) furnish the expression

$$
\begin{aligned}
\lim _{3 \mathrm{sh} \rightarrow 2 \mathrm{sh}}\left(G_{2, n}^{m}\right)= & \lim _{3 \mathrm{sh} \rightarrow 2 \mathrm{sh}}\left(G_{3, n}^{m}\right) \\
= & \sigma_{f}+\left(\sigma_{f}-\sigma_{b}\right)\left(I_{n}^{m}\left(f_{1}, s_{1}\right)+\frac{1}{S_{n}^{m}}-\frac{1}{F_{n}^{m}}\right) F_{n}^{m} \\
& +\left(\sigma_{b}-\sigma_{s}\right)\left(I_{n}^{m}\left(b_{1}, s_{1}\right)+\frac{1}{S_{n}^{m}}-\frac{1}{B_{n}^{m}}\right) B_{n}^{m} \\
& +\frac{\left(\sigma_{f}-\sigma_{b}\right)\left(\sigma_{b}-\sigma_{s}\right)}{\sigma_{b}} I_{n}^{m}\left(f_{1}, b_{1}\right)\left(I_{n}^{m}\left(b_{1}, s_{1}\right)+\frac{1}{S_{n}^{m}}-\frac{1}{B_{n}^{m}}\right) B_{n}^{m} F_{n}^{m} \\
= & G_{2, n}^{m},
\end{aligned}
$$


which proves the claim that $G_{2, n}^{m}$ is nothing else but the conductivity term for the two shells-model. Under these reductions (3.16), (3.17), and (3.18) immediately give the limits

$$
\begin{aligned}
& \lim _{3 \mathrm{sh} \rightarrow 2 \mathrm{sh}} u(\mathbf{r})=u_{2}(\mathbf{r})=g_{0}^{1} \frac{I_{0}^{1}(\rho)}{I_{0}^{1}\left(s_{1}\right)}+\sum_{n=1}^{\infty} \sum_{m=1}^{2 n+1} \frac{I_{n}^{m}(\rho)}{I_{n}^{m}\left(s_{1}\right)} \frac{1}{S_{n}^{m}} \frac{1}{G_{2, n}^{m}} \frac{\mathbf{Q} \cdot \nabla \mathbb{E}_{n}^{m}\left(\mathbf{r}_{0}\right)}{\gamma_{n}^{m}} \mathbb{E}_{n}^{m}(\rho, \mu, \nu), \\
& \lim _{3 \mathrm{sh} \rightarrow 2 \mathrm{sh}} u_{s}(\mathbf{r})=u_{2, s}(\mathbf{r})=u_{2}\left(s_{1}\right)+\sum_{n=1}^{\infty} \sum_{m=1}^{2 n+1} I_{n}^{m}\left(\rho, s_{1}\right) \frac{1}{G_{2, n}^{m}} \frac{\mathbf{Q} \cdot \nabla \mathbb{E}_{n}^{m}\left(\mathbf{r}_{0}\right)}{\gamma_{n}^{m}} \mathbb{E}_{n}^{m}(\rho, \mu, \nu), \\
& \lim _{3 \mathrm{sh} \rightarrow 2 \mathrm{sh}} u_{b}(\mathbf{r})=u_{2, b}(\mathbf{r})=u_{2, s}\left(b_{1}\right)+\sum_{n=1}^{\infty} \sum_{m=1}^{2 n+1} I_{n}^{m}\left(\rho, b_{1}\right) \frac{1}{\sigma_{b}} \frac{G_{1, n}^{m}}{G_{2, n}^{m}} \frac{\mathbf{Q} \cdot \nabla \mathbb{E}_{n}^{m}\left(\mathbf{r}_{0}\right)}{\gamma_{n}^{m}} \mathbb{E}_{n}^{m}(\rho, \mu, \nu),
\end{aligned}
$$

while (3.19) and (3.20) degenerate to

$$
\begin{aligned}
\lim _{3 \mathrm{sh} \rightarrow 2 \mathrm{sh}} u_{c}(\mathbf{r}) & =\lim _{3 \mathrm{sh} \rightarrow 2 \mathrm{sh}} u_{f}(\mathbf{r})=u_{2, f}(\mathbf{r}) \\
& =u_{2, b}\left(f_{1}\right)+\sum_{n=1}^{\infty} \sum_{m=1}^{2 n+1} I_{n}^{m}\left(\rho, f_{1}\right) \frac{1}{\sigma_{f}} \frac{\mathbf{Q} \cdot \nabla \mathbb{E}_{n}^{m}\left(\mathbf{r}_{0}\right)}{\gamma_{n}^{m}} \mathbb{E}_{n}^{m}(\rho, \mu, \nu) .
\end{aligned}
$$

Finally we draw our conclusions for the form of the potentials in the above four cases. The exterior potential for the case of the three confocal ellipsoidal shells, surrounding the homogeneous ellipsoidal brain, is given in (3.16). The exterior potential for the reduced cases of two shells, one shell as well as for the ellipsoidal homogeneous model is given by (4.24), (4.1), and (4.13), respectively. Observing these forms, it is important to note the role of the gradually reduced conductivity factors in each result. Similar, but more complicated results stand for the interior electric potentials, where all conductivity factors are involved and impose a certain effect on each potential.

\section{Geometrical degeneracies}

We consider here the reduction of the anisotropic ellipsoidal geometry to the isotropic spherical one [10]. We denote this by the symbol el $\rightarrow$ sr.

The case of four concentric spheres corresponds to el $\rightarrow$ sr, which implies the reduction.

$$
\lim _{\mathrm{el} \rightarrow \mathrm{sr}} s_{i}=s>b=\lim _{\mathrm{el} \rightarrow \mathrm{sr}} b_{i}>\lim _{\mathrm{el} \rightarrow \mathrm{sr}} f_{i}=f>c=\lim _{\mathrm{el} \rightarrow \mathrm{sr}} c_{i}, \quad i=1,2,3 .
$$

From the ellipsoidal system we obtain

$$
\lim _{\mathrm{el} \rightarrow \mathrm{sr}} h_{i}=0=\lim _{\mathrm{el} \rightarrow \mathrm{sr}} \mu=\lim _{\mathrm{el} \rightarrow \mathrm{sr}} \nu, \quad i=1,2,3,
$$


and finally, that

$$
\lim _{\mathrm{el} \rightarrow \mathrm{sr}} \rho=r .
$$

The elliptic integrals can be calculated now and they lead to the expressions

$$
\lim _{\mathrm{el} \rightarrow \mathrm{sr}} I_{n}^{m}(\rho)=\frac{1}{(2 n+1) r^{2 n+1}}
$$

for each $n=0,1,2, \ldots$ and $m=1,2, \ldots, 2 n+1$ and from the form of the Lamé functions [1] we can easily see that

$$
\lim _{\mathrm{el} \rightarrow \mathrm{sr}} E_{n}^{m}(\rho)=r^{n}
$$

In order to handle the indeterminacies, occurring during the reduction process, we use the following identities [1]

$$
\begin{aligned}
& \frac{\mathbb{E}_{2}^{2}(\rho, \mu, \nu)}{\Lambda_{s}^{\prime}-s_{1}^{2}}=\left(\Lambda_{s}^{\prime}-s_{2}^{2}\right)\left(x_{3}^{2}-x_{1}^{2}\right)+\left(\Lambda_{s}^{\prime}-s_{3}^{2}\right)\left(x_{2}^{2}-x_{1}^{2}\right)+\left(\Lambda_{s}^{\prime}-s_{2}^{2}\right)\left(\Lambda_{s}^{\prime}-s_{3}^{2}\right), \\
& \frac{\mathbb{E}_{2}^{2}(\rho, \mu, \nu)}{\Lambda_{s}^{\prime}-s_{2}^{2}}=\left(\Lambda_{s}^{\prime}-s_{1}^{2}\right)\left(x_{3}^{2}-x_{2}^{2}\right)+\left(\Lambda_{s}^{\prime}-s_{3}^{2}\right)\left(x_{1}^{2}-x_{2}^{2}\right)+\left(\Lambda_{s}^{\prime}-s_{1}^{2}\right)\left(\Lambda_{s}^{\prime}-s_{3}^{2}\right), \\
& \frac{\mathbb{E}_{2}^{2}(\rho, \mu, \nu)}{\Lambda_{s}^{\prime}-s_{3}^{2}}=\left(\Lambda_{s}^{\prime}-s_{1}^{2}\right)\left(x_{2}^{2}-x_{3}^{2}\right)+\left(\Lambda_{s}^{\prime}-s_{2}^{2}\right)\left(x_{1}^{2}-x_{3}^{2}\right)+\left(\Lambda_{s}^{\prime}-s_{1}^{2}\right)\left(\Lambda_{s}^{\prime}-s_{2}^{2}\right) .
\end{aligned}
$$

Also, some technical manipulations and the use of (5.6) lead to the key formula

$$
\lim _{\mathrm{el} \rightarrow \mathrm{sr}} \frac{\mathbb{E}_{2}^{2}(\mathbf{r})}{\left(\Lambda_{s}-\Lambda_{s}^{\prime}\right)\left(\Lambda_{s}^{\prime}-s_{i}^{2}\right)}=\frac{1}{2}\left(r^{2}-3 x_{i}^{2}\right), \quad i=1,2,3
$$

Replacing $\Lambda_{s}^{\prime}$ with $\Lambda_{s}$ and $\mathbb{E}_{2}^{2}(\rho, \mu, \nu)$ with $\mathbb{E}_{2}^{1}(\mathbf{r})$ in (5.6) we obtain the corresponding identities for $\mathbb{E}_{2}^{1}(\mathbf{r})$.Then using the Cartesian expressions of $\mathbb{E}_{n}^{m}$ and $E_{n}^{m}$, as they are given in [1], into the expressions (3.16) and (3.23), and taking the spherical limits (5.1)-(5.5) and (5.7) we arrive at

$$
\lim _{\mathrm{el} \rightarrow \mathrm{sr}} u(\mathbf{r})=g_{0}^{1} \frac{s}{r}+\frac{3}{4 \pi T_{1} r^{3}} \sum_{i=1}^{3} Q_{i} x_{i}-\frac{5}{8 \pi T_{2}} \frac{1}{r^{3}} \sum_{i=1}^{3} Q_{i} x_{0 i}+\frac{15}{8 \pi T_{2}} \frac{1}{r^{5}} \sum_{i, j=1}^{3} Q_{i} x_{0 j} x_{i} x_{j}+O\left(\frac{1}{r^{4}}\right),
$$


where

$$
\begin{aligned}
T_{1}= & \sigma_{c}+\left(\sigma_{f}-\sigma_{c}\right) \frac{2}{3}\left(1-\frac{c^{3}}{s^{3}}\right)-\left(\sigma_{f}-\sigma_{b}\right) \frac{2}{3}\left(1-\frac{f^{3}}{s^{3}}\right)-\left(\sigma_{b}-\sigma_{s}\right) \frac{2}{3}\left(1-\frac{b^{3}}{s^{3}}\right) \\
& +\frac{\left(\sigma_{f}-\sigma_{c}\right)\left(\sigma_{f}-\sigma_{b}\right)}{\sigma_{f}} \frac{2}{9}\left(1-\frac{c^{3}}{f^{3}}\right)\left(1-\frac{f^{3}}{s^{3}}\right) \\
& +\frac{\left(\sigma_{f}-\sigma_{c}\right)\left(\sigma_{b}-\sigma_{s}\right)}{\sigma_{b}} \frac{2}{9}\left(1-\frac{c^{3}}{b^{3}}\right)\left(1-\frac{b^{3}}{s^{3}}\right) \\
& -\frac{\left(\sigma_{f}-\sigma_{b}\right)\left(\sigma_{b}-\sigma_{s}\right)}{\sigma_{b}} \frac{2}{9}\left(1-\frac{f^{3}}{b^{3}}\right)\left(1-\frac{b^{3}}{s^{3}}\right) \\
& -\frac{\left(\sigma_{f}-\sigma_{c}\right)\left(\sigma_{f}-\sigma_{b}\right)\left(\sigma_{b}-\sigma_{s}\right)}{\sigma_{f} \sigma_{b}} \frac{2}{27}\left(1-\frac{c^{3}}{f^{3}}\right)\left(2+\frac{f^{3}}{b^{3}}\right)\left(1-\frac{b^{3}}{s^{3}}\right), \\
T_{2}= & \sigma_{c}+\left(\sigma_{f}-\sigma_{c}\right) \frac{3}{5}\left(1-\frac{c^{5}}{s^{5}}\right)-\left(\sigma_{f}-\sigma_{b}\right) \frac{3}{5}\left(1-\frac{f^{5}}{s^{5}}\right)-\left(\sigma_{b}-\sigma_{s}\right) \frac{3}{5}\left(1-\frac{b^{5}}{s^{5}}\right) \\
& +\frac{\left(\sigma_{f}-\sigma_{c}\right)\left(\sigma_{f}-\sigma_{b}\right)}{\sigma_{f}} \frac{6}{25}\left(1-\frac{c^{5}}{f^{5}}\right)\left(1-\frac{f^{5}}{s^{5}}\right) \\
& +\frac{\left(\sigma_{f}-\sigma_{c}\right)\left(\sigma_{b}-\sigma_{s}\right)}{\sigma_{b}} \frac{6}{25}\left(1-\frac{c^{5}}{b^{5}}\right)\left(1-\frac{b^{5}}{s^{5}}\right) \\
& -\frac{\left(\sigma_{f}-\sigma_{b}\right)\left(\sigma_{b}-\sigma_{s}\right)}{\sigma_{b}} \frac{6}{25}\left(1-\frac{f_{c}}{b^{5}}\right)\left(1-\frac{b^{5}}{s^{5}}\right) \\
& \frac{\left.\sigma_{f}-\sigma_{b}\right)\left(\sigma_{b}-\sigma_{s}\right)}{6}\left(1-\frac{c^{5}}{f^{5}}\right)\left(2+\frac{f^{5}}{b^{5}}\right)\left(1-\frac{b^{5}}{s^{5}}\right) . \\
& \\
& \left.\frac{6}{25}\right) \\
&
\end{aligned}
$$

Using the dyadic notation in the above expression we obtain the following compact form for the electric potential in the exterior of the layered spherical inhomogeneous conductor

$$
\lim _{\mathrm{el} \rightarrow \mathrm{sr}} u(\mathbf{r})=g_{0}^{1} \frac{s}{r}+\frac{3}{4 \pi T_{1}} \frac{\mathbf{Q} \cdot \hat{\mathbf{r}}}{r^{2}}-\frac{5}{8 \pi T_{2}} \mathbf{Q} \otimes \mathbf{r}_{0}: \frac{\tilde{\mathbf{I}}-3 \hat{\mathbf{r}} \otimes \hat{\mathbf{r}}}{r^{3}}+O\left(\frac{1}{r^{4}}\right) .
$$

Comparing the result (5.10) with the corresponding ellipsoidal one in (3.30) we appreciate the much higher level of complication exhibited by the ellipsoidal solution. In fact in each polyadic quantity, which carries the geometry and the physics of the conductor, the conductivity term varies in every multipole term. On the other hand, in the spherical case, the conductivity term changes only by the order $n$ of the corresponding multipole term, reflecting in this way the geometrical simplicity of the spherical model.

It is further worth pointing out, the similarity of the exterior potential for the three shells spherical model, as given in (5.10) with the corresponding result of the simple spherical one [8]

$$
u_{\mathrm{sr}}(\mathbf{r})=g_{0}^{1} \frac{s}{r}+\frac{3}{4 \pi \sigma} \frac{\mathbf{Q} \cdot \hat{\mathbf{r}}}{r^{2}}-\frac{5}{8 \pi \sigma} \mathbf{Q} \otimes \mathbf{r}_{0}: \frac{\tilde{\mathbf{I}}-3 \hat{\mathbf{r}} \otimes \hat{\mathbf{r}}}{r^{3}}+O\left(\frac{1}{r^{4}}\right) .
$$


We see that both potentials enjoy exactly the same form with the only difference that the conductivity factors $T_{1}^{-1}, T_{2}^{-1}$ are reduced to the constant $\sigma^{-1}$ for the homogeneous model. The fact that the conductivity factors in (5.10) incorporate the boundaries of the conductivity supports, thus making the conductor "visible" in every term of (5.10), illustrates the strong influence that the shells have upon the electric field. On the other hand, in (5.11) the boundary of the conductor appears only on the leading monopole term, which also carries the arbitrary constant $g_{0}^{1}$.

\section{Acknowledgments}

The first author acknowledges support from the postgraduate scholarship of the "Onassis Foundation." This work was performed during the sabbatical year 2003-2004 of the second author in the Division of Applied Mathematics of the Department of Chemical Engineering in the University of Patras.

\section{References}

[1] G. Dassios and F. Kariotou, Magnetoencephalography in ellipsoidal geometry, Journal of Mathematical Physics 44 (2003), no. 1, 220-241.

[2] J. C. de Munck, The potential distribution in a layered anisotropic spheroidal volume conductor, Journal of Applied Physics 64 (1988), no. 2, 464-470.

[3] A. S. Fokas, I. M. Gelfand, and Y. Kurylev, Inversion method for magnetoencephalography, Inverse Problems. An International Journal on the Theory and Practice of Inverse Problems, Inverse Methods and Computerized Inversion of Data 12 (1996), no. 3, L9-L11.

[4] M. S. Hämäläinen, R. Hari, R. J. Ilmoniemi, J. Knuutila, and O. Lounasmaa, Magnetoencephalography - theory, instrumentation, and applications to noninvasive studies of the working human brain, Reviews of Modern Physics 65 (1993), no. 2, 413-497.

[5] E. W. Hobson, The Theory of Spherical and Ellipsoidal Harmonics, Chelsea, New York, 1955.

[6] R. J. Ilmoniemi, M. S. Hämäläinen, and J. Knuutila, The forward and inverse problems in the spherical model, Biomagnetism: Applications and Theory (H. Weinberg, G. Stroink, and T. Katila, eds.), Pergamon Press, New York, 1985, pp. 278-282.

[7] G. Kamvyssas and F. Kariotou, The effect of an ellipsoidal shell on the direct EEG problem, to appear in Bulletin of the Greek Mathematical Society, 2004.

[8] F. Kariotou, Electroencephalography in ellipsoidal geometry, Journal of Mathematical Analysis and Applications 290 (2004), no. 1, 324-342.

[9] J. Malmivuo and R. Plonsey, Bioelectromagnetism-Principles and Applications of Bioelectric and Biomagnetic Fields, vol. 461, Oxford University Press, New York, 1995.

[10] J. Malmivuo, V. Suihko, and H. Eskola, Sensitivity distributions of EEG and MEG measurements, IEEE Transactions on Biomedical Engineering 44 (1997), no. 3, 196-208.

[11] T. Miloh, Forces and moments on a tri-axial ellipsoid in potential flow, Israel Journal of Technology 11 (1973), no. 1-2, 63-74.

[12] G. Nolte, T. Fieseler, and G. Curio, Perturbative analytical solutions of the magnetic forward problem for realistic volume conductors, Journal of Applied Physics 89 (2001), no. 4, 2360-2369.

[13] J. Sarvas, Basic mathematical and electromagnetic concepts of the biomagnetic inverse problem, Physics in Medicine and Biology 32 (1987), no. 1, 11-22.

[14] W. S. Snyder, M. R. Ford, G. G. Warner, and H. L. Fisher Jr., Estimates of absorbed fractions for monoenergetic photon sources uniformly distributed in various organs of a heterogeneous phantom, Journal of nuclear medicine 10 (1969), pamphlet no. 5, revised 1978, supplement no. 3.

[15] A. Sommerfeld, Electrodynamics. Lectures on Theoretical Physics, Vol. III, Academic Press, New York, 1952. 
18 The complete ellipsoidal shell-model in EEG imaging

[16] W. X. Wang, The potential for a homogeneous spheroid in a spheroidal coordinate system. I. At an exterior point, Journal of Physics. A. Mathematical and General 21 (1988), no. 22, 4245-4250.

S. N. Giapalaki: Department of Chemical Engineering, University of Patras, 26504 Patras, Greece E-mail address: sonia@rea.chemeng.upatras.gr

F. Kariotou: Hellenic Open University, 16, Sahtouri Street \& Ag. Andreou Street, 26222 Patras, Greece

Current address: Department of Chemical Engineering, University of Patras, 26504 Patras, Greece

E-mail address: fkario@rea.chemeng.upatras.gr 


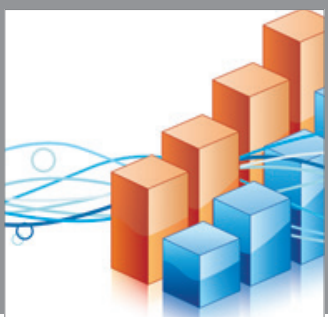

Advances in

Operations Research

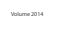

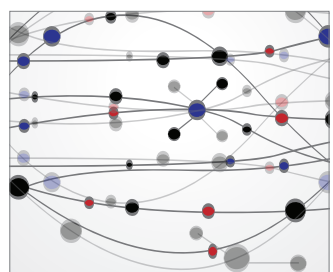

\section{The Scientific} World Journal
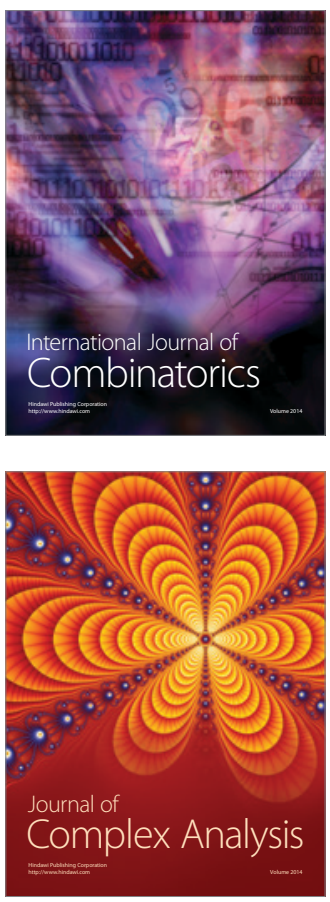

International Journal of

Mathematics and

Mathematical

Sciences
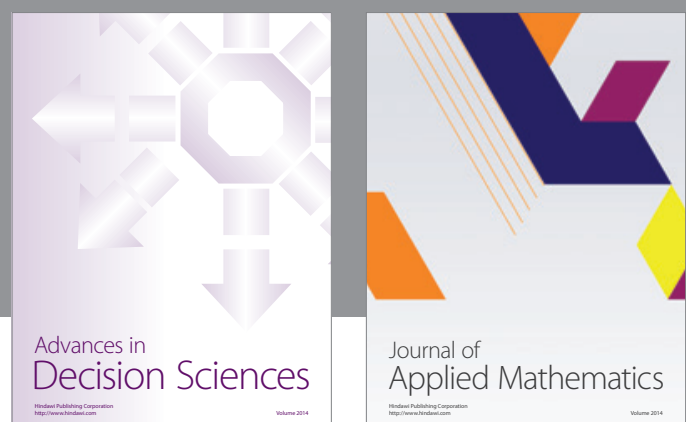

Journal of

Applied Mathematics
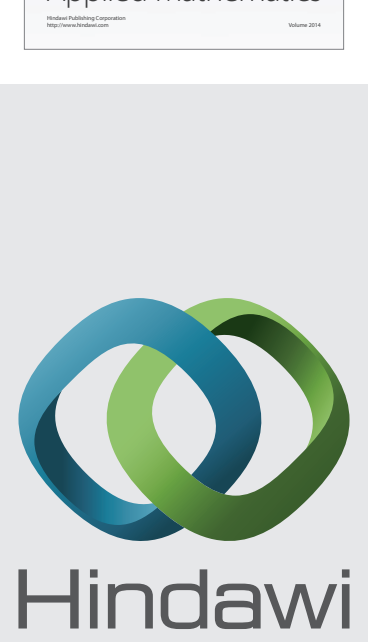

Submit your manuscripts at http://www.hindawi.com
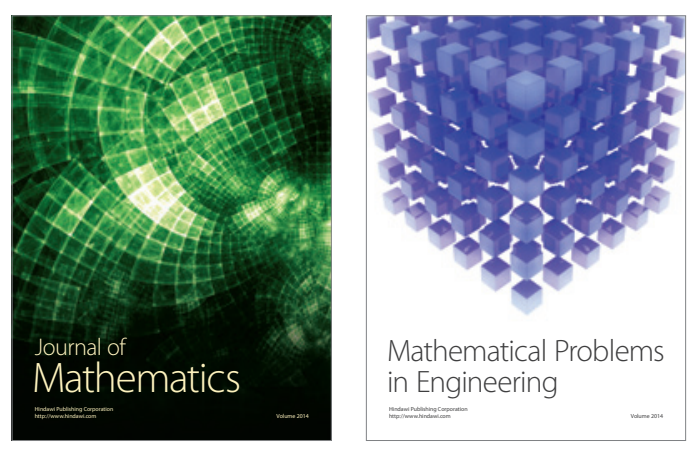

Mathematical Problems in Engineering
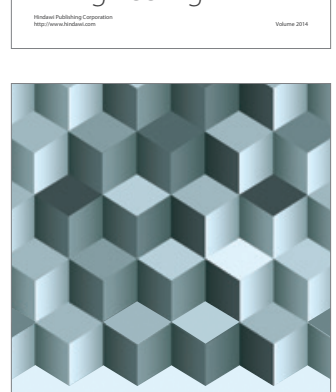

Journal of

Function Spaces
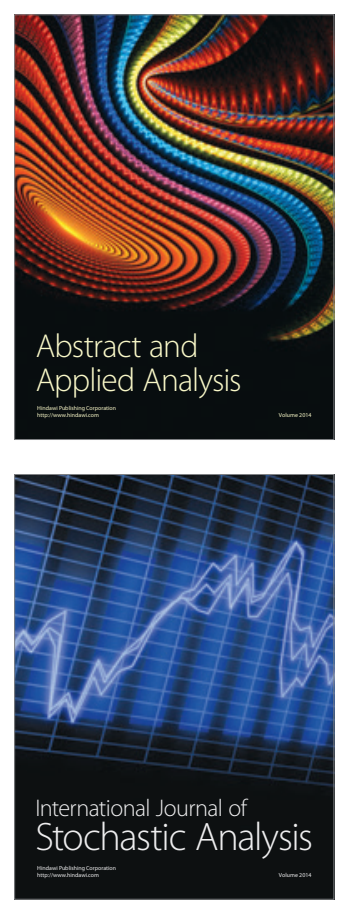

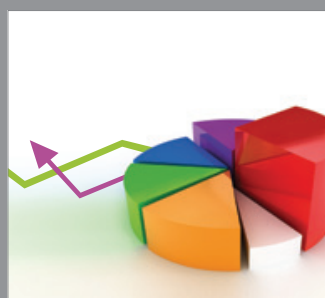

ournal of

Probability and Statistics

Promensencen
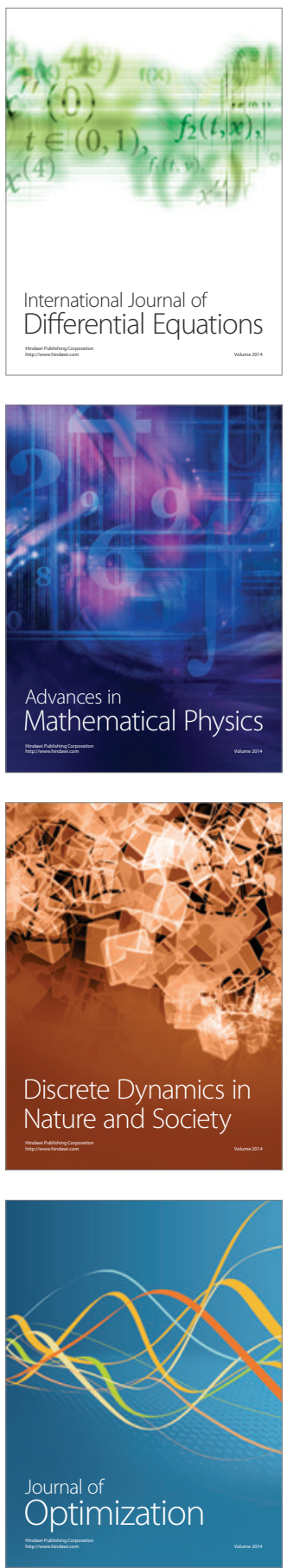